\title{
Metastatic Basal Cell Carcinoma to the Parotid Gland
}

\author{
Pascalino B Romeo, $M D^{1 *}$, Fraser Donaldson, $M B B S^{1}$, Ibrahim M Zardawi, FRCPA ${ }^{2}$ and \\ Kristian Sørensen, MBChB (Hons) MRCS FRCS (Plast) ${ }^{1}$ \\ ${ }^{1}$ Department of Plastic Surgery, Cairns Base Hospital, Australia \\ ${ }^{2}$ Department of Anatomical Pathology, Cairns Base Hospital, Australia
}

\begin{abstract}
Summary
Basal cell carcinoma is a very common skin cancer in Australia. It is a locally invasive neoplasm which can rarely have life threatening effects. There have been many reported cases of metastatic BCC though it is uncommon, rarer still is spread to the parotid. We present a case of a patient in Far North Queensland who had a BCC metastasize to his parotid from a close by primary.
\end{abstract}

\section{Keywords}

Basal cell carcinoma, Skin cancer pathology, Parotid gland

\section{Introduction}

Basal Cell Carcinoma (BCC) is the most common invasive skin neoplasm in Australia. It carries with it a lifetime development risk of $30 \%$ and accounts for approximately $70 \%$ of skin cancers Australia wide [1]. BCC is predominantly found in the head and neck region. Reports in the literature state $85 \%$ of metastatic BCC will originate in the head and neck [2] as up to $65 \%$ of all BCCs arise on the face or head [3]. There have been case reports of $\mathrm{BCC}$ metastasizing however it is rare with the documented incidence being somewhere between $0.008 \%$ and $0.5 \%$ [4]. A review of 850 cases of metastatic BCC concluded that facial BCC with a greater than $3 \mathrm{~cm}$ diameter had a $1.9 \%$ incidence of metastasis [3]. There have been two documented cases of BCC metastasis to the parotid gland [5], here we present a third with metastasis to the parotid gland and intra-parotid lymph nodes.

\section{Case Report}

An 81-year-old otherwise very healthy male was referred to the Plastic Surgery clinic with a carcinoma ex plemorphic adenoma of the left parotid gland. The patient had a history of multiple non-melanoma skin cancers which he had occasionally treated with Black Salve. He had noticed the lump and had it investigated by his GP. FNA of the lesion showed a poorly differentiated carcinoma which was presumed to have originated as a pleomorphic ademona. At clinic it was noted that the patient had a clinical BCC $1 \mathrm{~cm}$ anterior to his helical root. The patient underwent staging scans (Figure 1) and discussion at the regional MDT meeting at the Royal Brisbane and Women's Hospital (RBWH). The recommendation was for a superficial parotidectomy and upper neck dissection and post-operative radiation. The pre-auricular BCC was also

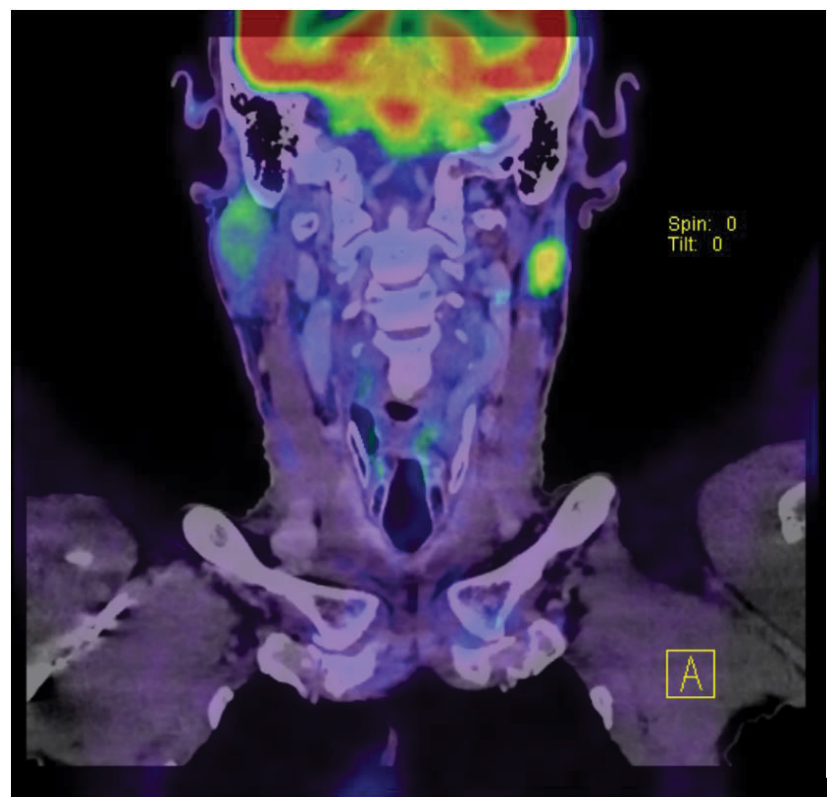

Figure 1: Staging PET scan showing increased uptake in the parotid gland.

*Corresponding author: Pascalino B Romeo, MD, Department of Plastic Surgery, Cairns Base Hospital, Australia

Accepted: December 29, 2021

Published online: December 31, 2021

Citation: Romeo PB, Donaldson F, Zardawi IM, et al. (2021) Metastatic Basal Cell Carcinoma to the Parotid Gland. J Head Neck Surg 3(2):163-165

Copyright: ( 2021 Romeo PB, et al. This is an open-access article distributed under the terms of the Creative Commons Attribution License, which permits unrestricted use, distribution, and reproduction in any medium, provided the original author and source are credited. 
excised opportunistically. The procedure was uneventful and the patient made an excellent recovery with minimal facial weakness and no complications.

Pathological evaluation of the specimens was undertaken initially at the Cairns Base Hospital where the patient was seen and subsequently at the tertiary referral hospital in Brisbane, Queensland. The pre-auricular lesion showed a 14 $\mathrm{mm}$ diameter lesion that was a $6 \mathrm{~mm}$ thick nodular basal cell carcinoma on initial evaluation. The parotid tumour was 20 $\mathrm{mm}$ in diameter with an infiltrative pattern, perineuroal and lymphovascular invasion and a focally myxoid stroma. Initial evaluation indicated carcinoma ex pleomorphic adenoma and further immunostains were done. The parotid tumour cells were strongly CK5/6 positive and Myoepithelial markers SMA and P63 were also positive. The case was discussed at the skin cancer MDT and referred for second opinion at the state tertiary referral centre department of anatomical pathology. The features of the tumour were consistent with a moderately differentiated basaloid malignant tumour. The parotid tumour was compared with the per-auricular tumour. Interestingly both tumours demonstrate similar staining pattern with positive staining with p63, ck14, bcl2 and BerEp4 (Figure 2). It was concluded that both tumours shared the same histological and immunohistochemistry pattern and that the parotid tumour was a deposit of metastatic BCC likely from the pre-auricular primary. The patient underwent postoperative radiation to the parotid bed and the site of the preauricular primary.

\section{Discussion}

BCC is the most common skin malignancy but thankfully it has a very low rate of metastasis. There have been attempts to identify prognostic features of the primary BCC which may indicate the capacity to metastasize. There are several varieties of BCC including nodular and micronodular, infiltrative and morphoeic subtypes which are more locally aggressive and harder to primarily excise [6]. Histopthologically, the nodular subtype is the most common characterised by nodular nests of basaloid cells with myxoid change and peripheral palisading of cells [7]. Morphoeic and infiltrative carcinomas will have thick columns of basaloid cells in a collagenous stroma. Higher mitotic rates and more necrosis confer a higher rate of invasion into the deeper dermis, adjacent structures as well as perineural invasion [7]. The primary tumour in this case was of the nodular subtype however the tumour itself was 6 $\mathrm{mm}$ thick with no other high-risk pathological features which in itself is unusual. Its clinical location however is high-risk. High-risk features such as infiltrative subtype and size $>3 \mathrm{~cm}$ have been shows to increase rate of metastasis in BCC [4]. There have been attempts to isolate immunohostochemical markers [4] which may indicate the likelihood of a BCC to metastasize however there is no clear consensus yet. A review of 6 cases of metastatic BCC postulated that the propensity of $B C C$ to metastasize from the head and neck region may be due to the thin skin of the area and the high concentration of large calibre blood vessels [4].

Another high-risk feature of $\mathrm{BCC}^{\prime} \mathrm{s}$ is the presence of perineural or lymphovascular invasion. These features have been associated with an increased rate of local recurrence [8]. Recurrence has been shown by all published series on the surgical excision of BCC to be a risk factor for inferior cure rates when compared to primary BCC [6]. There has been no indication that recurrence may increase the rate of metastasis of BCC. This patient had treated undiagnosed skin cancers in the past with Black Salve, a topical agent with no evidence base for its efficacy. These authors have found anecdotally in Far North Queensland that patients who advocate the use of this chemical often have more advanced or aggressive disease. Whether this is a product of the chemical itself or due to the time delay in treatment is unclear.
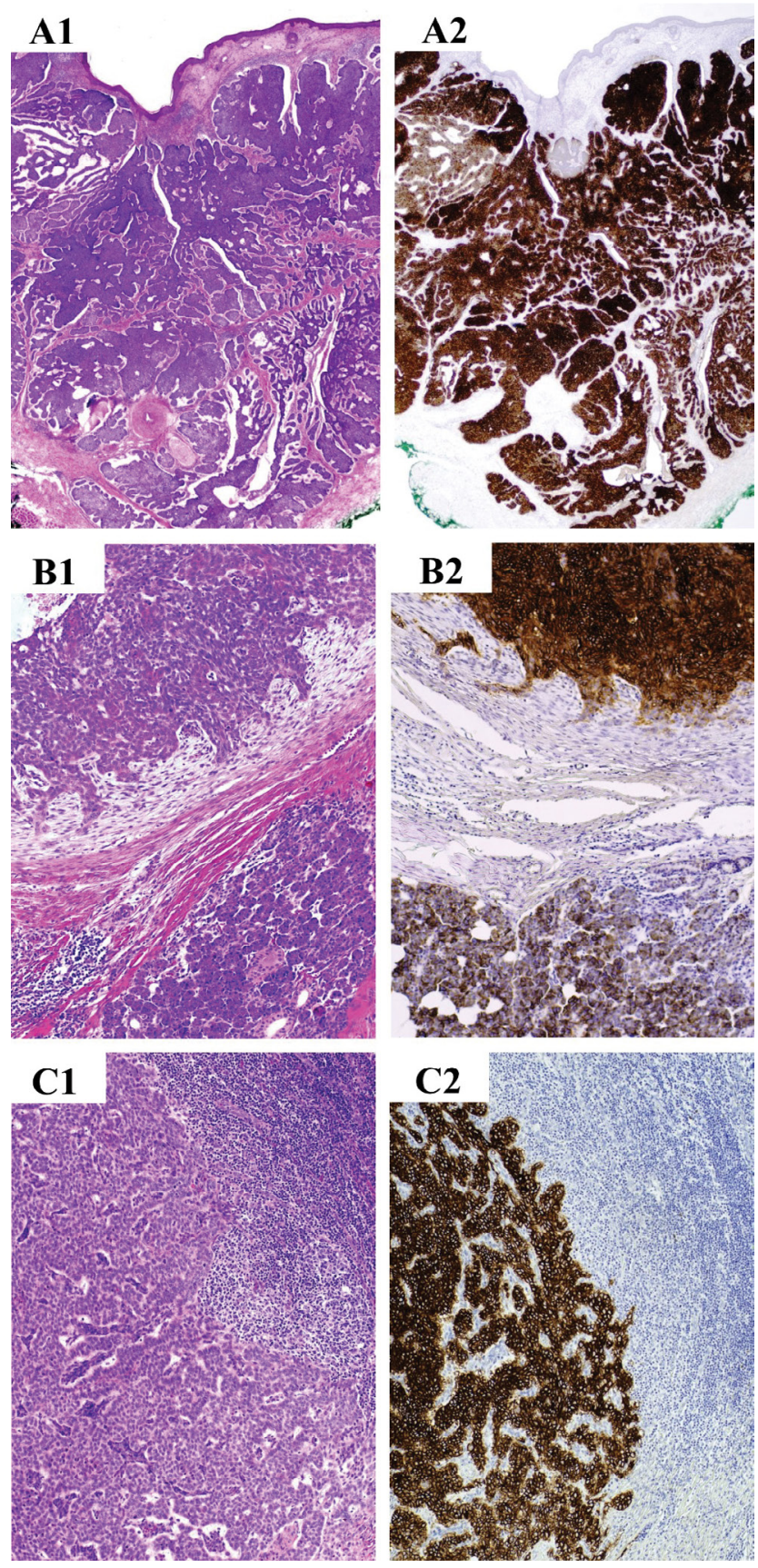

Figure 2: The parotid tumor was compared with the per-auricular tumor. 


\section{Conclusions}

This case of primary nodular BCC metastasizing to the parotid highlights the possibility that pathologically low risk tumours may undergo metastasis. Most cases in the literature have had high risk features including size and histological subtype.

\section{Authors Contribution}

All authors equally contribution to this paper.

\section{Conflicts of Interest}

None.

\section{References}

1. Lear JT, Smith A (1997) Basal cell carcinoma. Postgrad Med J 73: 538-542.
2. Neuenschwander MC, Pribitkin EA, Sataloff RT (2000) Botulinum toxin in otolaryngology: A review of its actions and opportunities for use. Ear Nose Throat J 79: 788-789.

3. Snow SN, Sahl Lo WJS, Mohs FE, et al. (1994) Metastatic basal cell carcinoma. Report of five cases. Cancer. Cancer 73: 328-335.

4. Lau J, Guminski A, Gill A, et al. (2018) Metastatic basal cell carcinoma: A review of six cases. Australian J Otolaryngol 1: 20.

5. Kurian R, Di Palma S, Barrett A (2014) Basal cell carcinoma metastatic to the parotid. Head Neck Pathol 8: 349-353.

6. Telfer NR, Colver GB, Morton CA (2008) Guidelines for the management of basal cell carcinoma. Br J Dermatol 159: 35-48.

7. Crowson A (2006) Basal cell carcinoma: Biology, morphology and clinical implications. Mod Pathol 19: S127-S147.

8. Ting PT, Kasper R, Arlette JP (2009) Metastatic basal cell carcinoma: Report of two cases and literature review. J Cutan Med Surg 9: 10-15. 